\title{
Facies Classification in 3D Seismic Data Volume of the Brazilian Pre-salt through Convolutional Neural Networks Technology
}

Igor Braga, Igor Barbosa, João Puga, Anderson Franco, Luciano Dias Pereira and Geovane Ouverney, Invision Geophysics

Copyright 2019, SBGf - Sociedade Brasileira de Geofísica

This paper was prepared for presentation during the $16^{\text {th }}$ International Congress of the Brazilian Geophysical Society held in Rio de Janeiro, Brazil, 19-22 August 2019.

Contents of this paper were reviewed by the Technical Committee of the $16^{\text {th }}$ International Congress of the Brazilian Geophysical Society and do not necessarily represent any position of the SBGf, its officers or members. Electronic reproduction or storage of any part of this paper for commercial purposes without the written consent of the Brazilian Geophysical Society is prohibited.

\section{Abstract}

Carbonate reservoirs became the center of attention for the Brazilian offshore oil industry after the oil discoveries in the Santos Pre-salt layer, motivating efforts to development of new methods to characterization of these rocks. Artificial neural network structures were developed based on the human brain. The processing units, called artificial neurons, are simplified models of biological neurons. In this same sense, convolutional neural networks were elaborated to serve as the computer vision. Despite the potential of this technology, the convolutional networks have not been successfully adapted to work with seismic data. The main goal of this work is to develop a new methodology, focusing in data preparation; conditioning and attribute generation, to perform facies classification through convolutional neural networks technology in 3D seismic data volume, concentrating in characterize oil reservoir zones in Brazilian Pre-salt interval. Using this new methodology, the 2-Facies model was generated, classifying oil reservoir zones from Pre-salt seismic data providing a valuable information about lateral distribution of these reservoirs and allowing to identify new targets in the area of interest.

\section{Introduction}

Since the huge discoveries of Brazilian Pre-salt reservoirs in Santos basin, it has been concentrating efforts to characterize and describe the complexity of these carbonate reservoirs. The pore structure and variations in reservoir quality are very difficult to predict, especially from seismic data. The subtle facies changes, low seismic expression of internal limits and velocity dispersion are characteristics that require a more elaborate model of data processing and facies classification, inspiring the development of new methods including over last years the artificial intelligence.

Thought and learning are carried out by the human brain from the processing of information. The main cell of the human nervous system is the neuron and its basic function is to drive impulses under certain operating conditions (Da Silva et al., 2017). Artificial neural network structures were developed based on the human brain. The processing units, called artificial neurons, are simplified models of biological neurons. These models were inspired from the analysis of how a cell membrane of a neuron generates and propagates electrical impulses (Hodgkin and Huxley, 1990).

McCulloch and Pitts (1943) proposed the most used neuron model in different artificial neural network architectures, as can be seen in Figure 1. The artificial neuron is composed basically of seven elements: input signals $(x 1, x 2, \ldots, x n)$; synaptic weights $(w 1, w 2, \ldots, w n)$; linear aggregator $(\Sigma)$; activation threshold or bias $(\theta)$; activation potential (u); activation function (g) and the output signal $(y)$ that consists on the final value produced by the neuron (Da Silva et al., 2017).

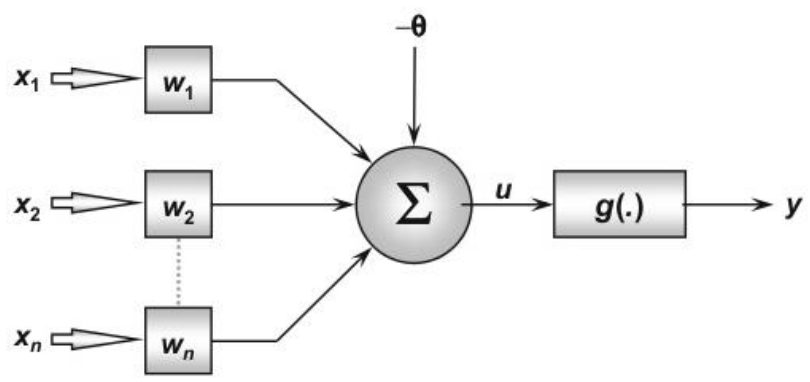

Figure 1 - Artificial neural. Source: Da Silva et al., 2017

The Convolutional Neural Networks (CNNs) also known as convolutional networks (LeCun, 1989) are a specialized kind of neural network that use convolution in place of general matrix multiplication for processing data that has a known grid-like topology.

Convolutional networks have been tremendously successful in practical applications (Goodfellow et al., 2016). Despite the potential of this technology, the convolutional networks have not yet been successfully adapted to work with seismic data. Figure 2 presents a simple model of convolutional neural network.

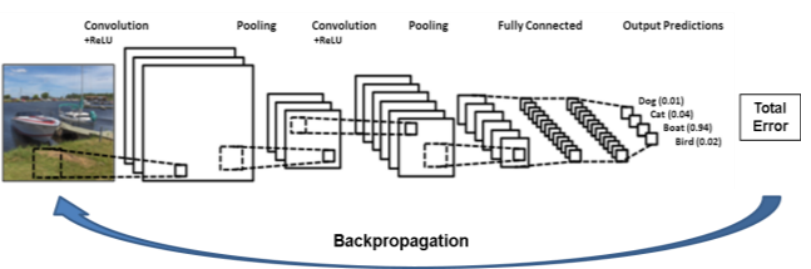

Figure 2 - A simple CNN model. Source: Modified of Clarifai, accessed on November 27, 2017

The convolution and pooling layers act as feature extractors from the input image while the fully connected layer acts as a classifier. First it initializes all filters, parameters and weights with random values; then, the network takes a training image as input, goes through the forward propagation step (convolution, ReLU and pooling 
operations along with forward propagation in the fully connected layer) and finds the output probabilities for each class, but since the weights are randomly assigned for the first training example, output probabilities are also random. Afterwards, it calculates the total error at the output layer and use backpropagation to estimate the gradients of the error with respect to all weights in the network and use gradient descent to update all filter values and parameters to minimize the output error; finally the training process repeats itself with all images in the training dataset until the output error reaches a predetermined minimum value.

The purpose of this work is to capitalize on the special preparation of input data by combining two successful approaches: an advanced data preparation workflow to provide high-resolution images and the meta-attribute concept to create a new methodology, with the objective of separates oil reservoirs from no reservoir zones in 3D seismic data volume of the Brazilian Pre-salt through convolutional neural networks technology.

\section{Method}

The methodology developed in this work involves several steps, as shown in the full workflow of facies classification present in Figure 3.
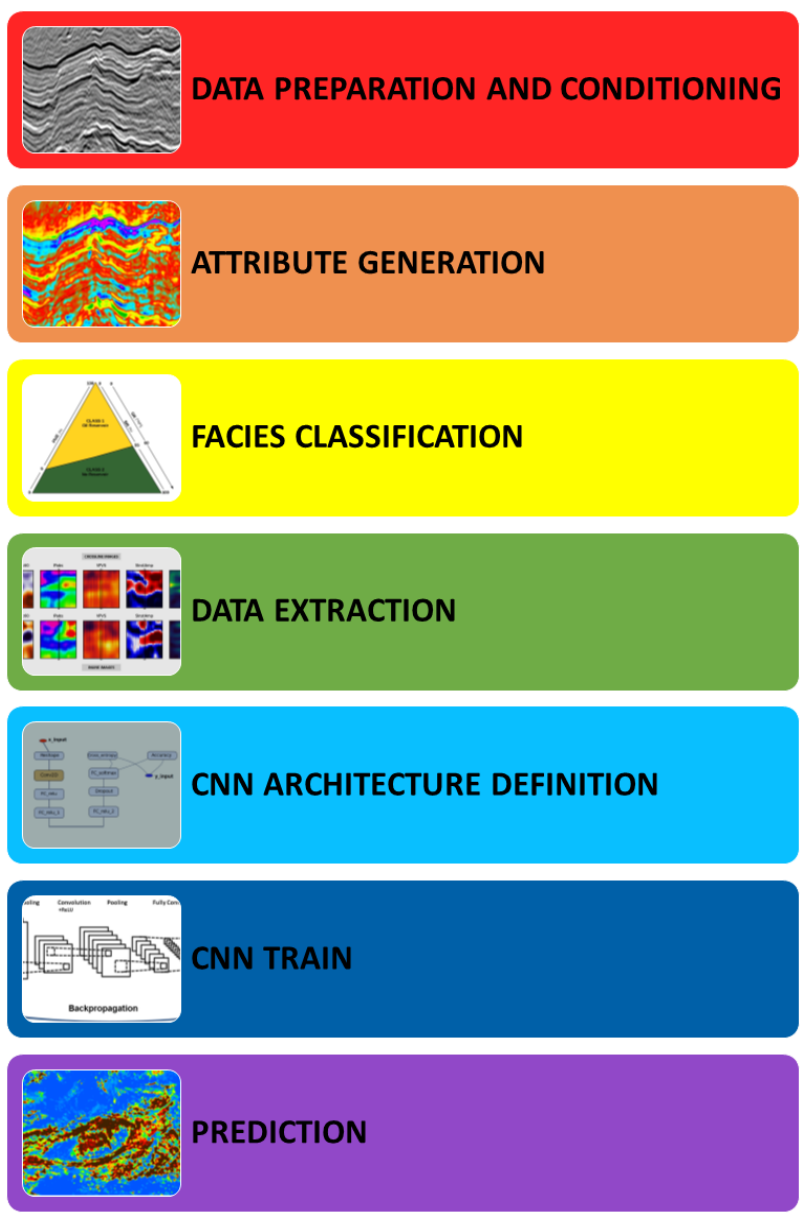

Figure 3-Full workflow of the new methodology of facies classification from 3D seismic data using CNN technology
The data required to develop this workflow comprise of a 3D seismic data and several wells with their respective logs from the Brazilian Pre-salt interval.

This work begins with data preparation and conditioning, which aims to attenuate noise and increasing the spectral content of seismic data, achieving better seismic image, attributes and inversion results.

The Figure 4 shows a common image gather after each step of the pre-stack seismic data conditioning. First, an angle mute above $40^{\circ}$ was applied to eliminate areas with high stretching. Following, the MultiFilt was employed, a filter that uses curvelet transform to perform seismic data decomposition, allowing noise attenuation. The WRMO, wavelet residual moveout correction, was designed to correct misalignments of the seismic reflections, an important step for AVO analysis. Afterwards, the InvQ routine (Braga, 2011; Braga and Moraes, 2013) was applied to raise the amplitude of the high frequency components of the signal, compensating absorption and dispersion effects.

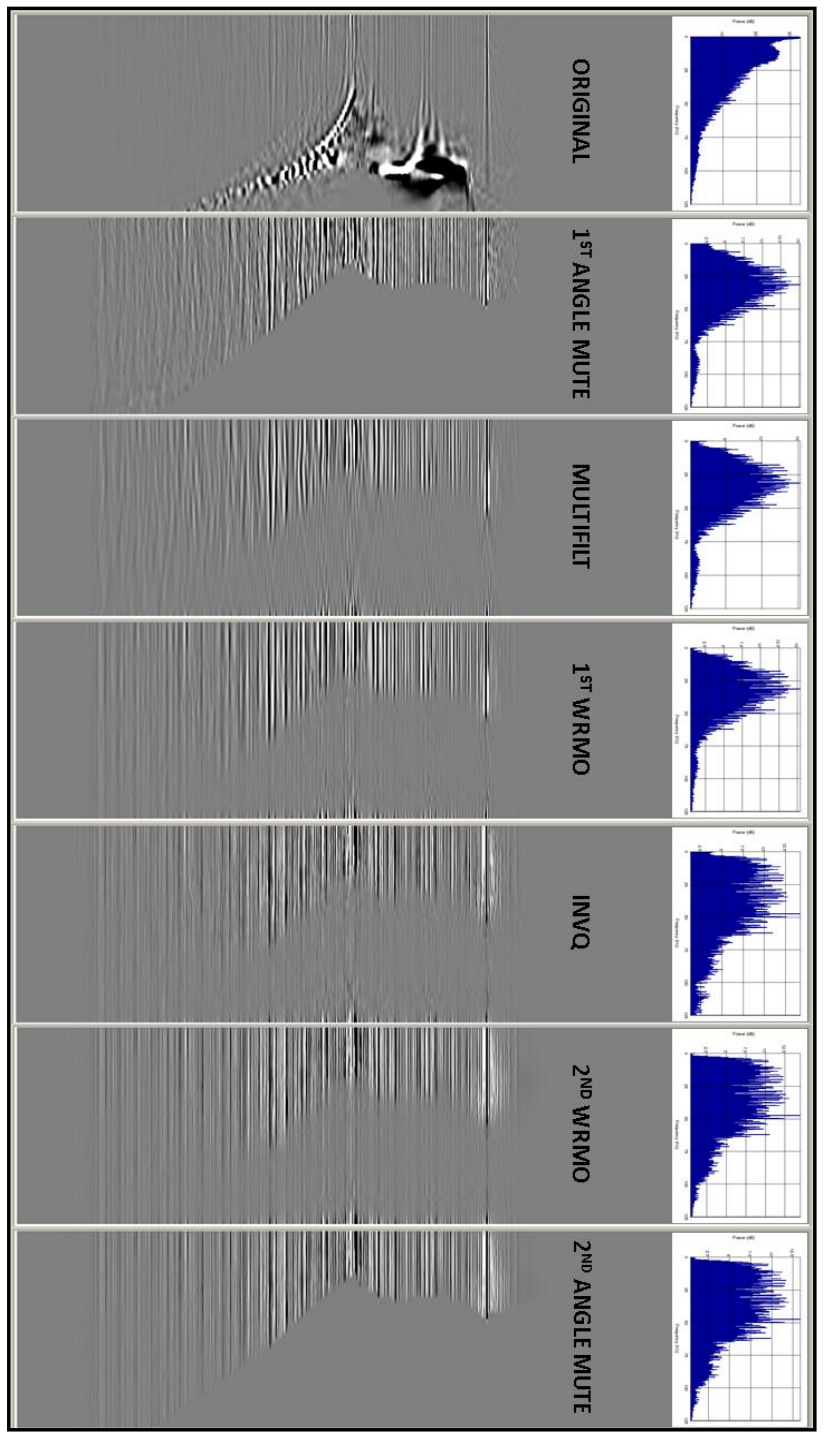

Figure 4 - Common image gather after each step of the pre-stack seismic data conditioning 
The Attribute generation is performed aiming to provide useful information for quantitative and qualitative seismic interpretation as explained in the schematic diagram presented in Figure 5. These different views of seismic data generally reveal expressions of geological features, such as faults, stratigraphic units and facies variation, helping to unravel the depositional environment and structural deformation of the study area.

Concerning to Pre-salt interval, the best results were achieved applying elastic attributes (absolute $P$ impedance and VP/VS ratio); frequency-based attributes (instantaneous spectral decomposition of $60 \mathrm{~Hz}$ and instantaneous amplitude) and texture-based attribute (GLCM standard deviation).
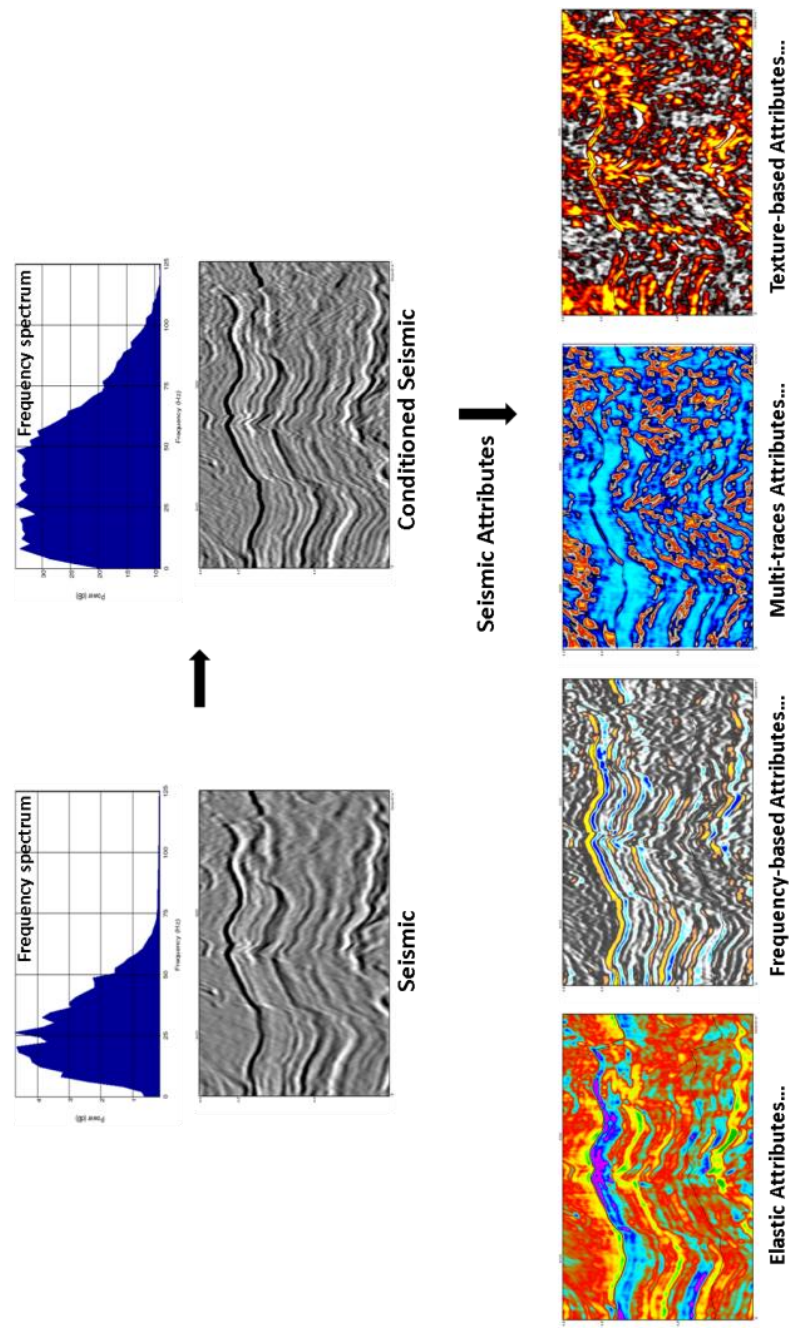

Figure 5 - Generation of seismic attributes from conditioned seismic data

The facies classification was based on well attribute logs located in the area of interest, considering the seismic features. The main goal was to define a 2 -Facies model that separates oil reservoirs zones from no reservoir zones. For this approach were used the following well logs: Gamma ray (GR), Saturation of Water (SW) and Effective Porosity (PhiE). A diagram illustrating the 2Facies model and the cutoff limits by well logs are shown in Figure 6.
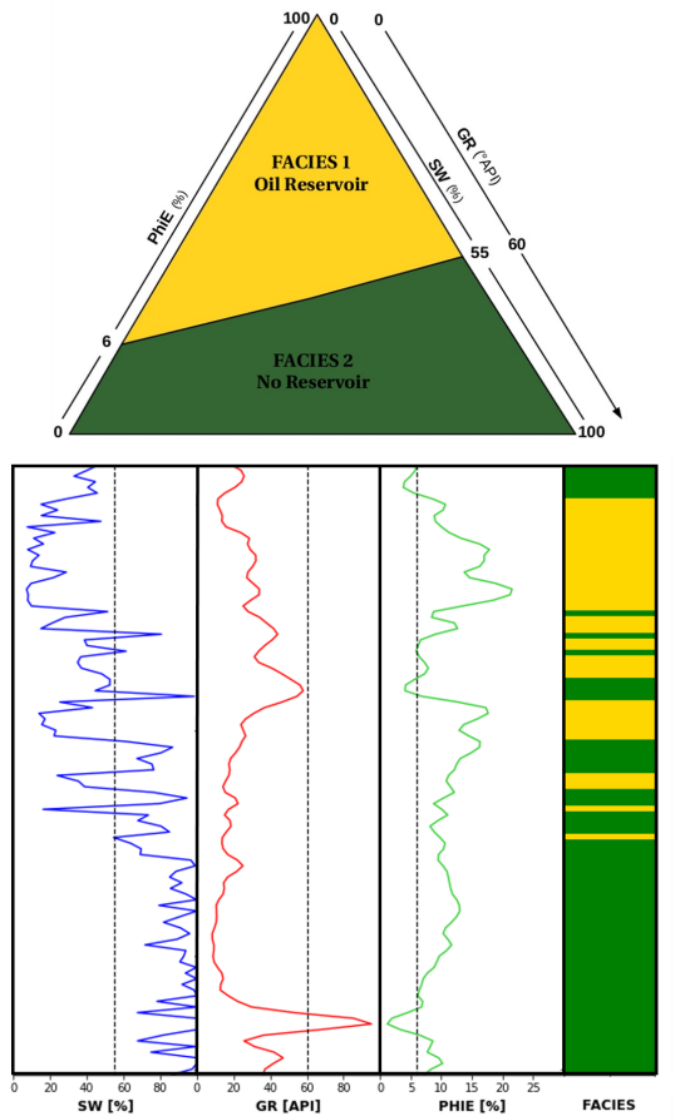

Figure 6 - 2-Facies model diagram. Facies 1 (yellow) oil reservoir facies, including effective porosity above $6 \%$, saturation of water lower than 55\% and gamma ray lower than $60^{\circ} \mathrm{API}$

The data extraction uses as input the logs generated during facies classification and the seismic attributes. An extractor (Figure 7) runs the seismic volumes marked by the track of each well collecting small seismic sections and assigns the corresponding label found in the facies classification log.

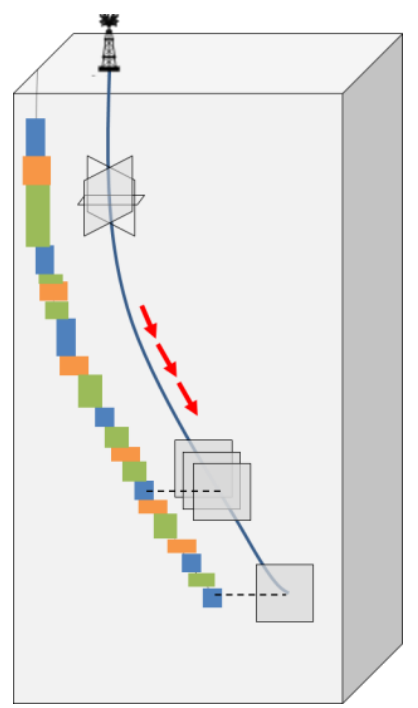

Figure 7-Schematic model of the well extractor 
The seismic data attributes were extracted in the inline and crossline directions (the network was fixed considering the image size of $25 \times 25$ samples), to be possible predict in both ways, generating a more accurately classified volume. The centerline of the images extracted in the inline and crossline views are the same, since they follow the well's track (Figure 8).

As mentioned before, five seismic attributes were used to compose the dataset: two frequency-based attributes (instantaneous spectral decomposition of $60 \mathrm{~Hz}-$ InstSpecDec60 and instantaneous amplitude SInstAmp), two elastic attributes (absolute P impedance IPabs and VP/VS ratio - VPVS) and one texture-based attribute, the GLCM standard deviation - StdDev.

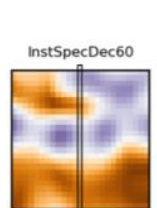

InstSpecDec60
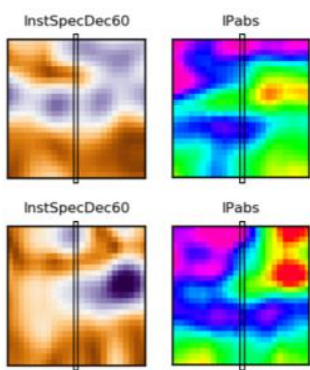

IPabs
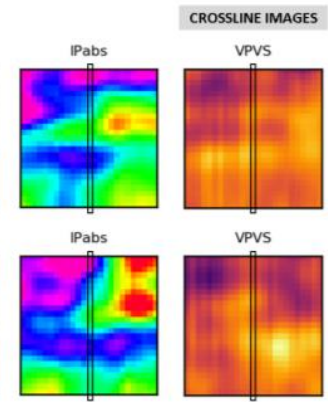

vPvs

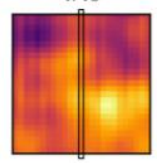

INUINE IMAGES
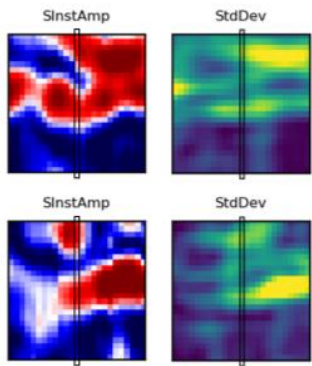

StdDev

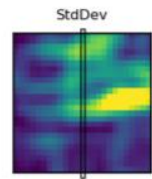

Figure 8 - Images extracted from the same point in the inline and crossline directions for five different attributes

The CNN architecture used to perform the training and prediction was calibrated through parametrization tests. The CNN graph (Figure 9) consists of a convolutional layer with a $2 \times 2$ kernel and 64 features, followed by three fully connected layers with 64 neurons and ReLU activator and a dropout layer followed by a fully connected final layer with softmax activation function, which makes the prediction.

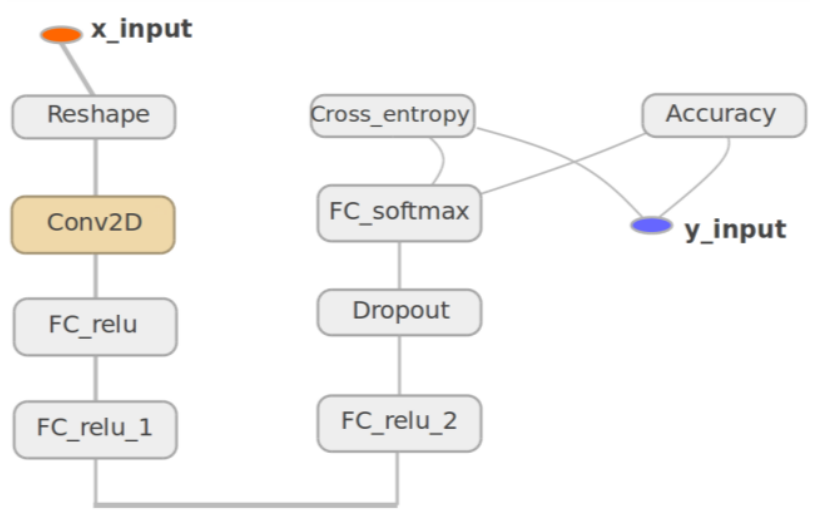

Figure 9-CNN graph

The prediction is the last stage in the CNN facies classification workflow. After seismic data preparation and conditioning, attribute generation, facies classification, data extraction, CNN architecture definition and CNN train, the output volume generated by prediction aims to classify the input seismic data into different facies, according to the model used previously. In this work, it is used the 2-Facies model described before.

\section{Results}

Taking the Brazilian Pre-salt reservoirs heterogeneities into consideration, this new methodology developed using the convolutional neural network technology, reached relevant results in the characterization of oil reservoir seismic facies.

Figure $10 \mathrm{a}$ presents a random line of the original 3D seismic data volume of the Brazilian Pre-salt. On the other hand, Figure 10b shows the conditioned seismic data, after data preparation and conditioning - this step was crucial in increasing the frequency bandwidth of the seismic data, improving the resolution of events and consequently providing better-input images to the CNN. Figure $10 \mathrm{c}$ presents the 2-Facies model prediction at the random line that cross two wells from Pre-Salt layer - the well logs in Figure 10 are that generated during facies classification, as described in Figure 6.

In addition, Figure $10 \mathrm{~d}$ shows the prediction results over the conditioned seismic highlighting the consistency between the seismic data, the CNN results and the well logs, and lastly, Figure 10e highlights the Pre-salt layer where is possible to observe the main faults defining structural highs (more expressive as oil reservoirs facies according to the classification) and structural lows.

The average accuracy reached to the case of all wells presented to CNN was $98 \%$, an extraordinarily high value that ensures that facies classification using convolutional neural network technology is a safe method for the purpose of this work.

\section{Conclusions}

In this work, it was successfully developed and applied a new methodology, focusing in data preparation, conditioning and attribute generation, to perform facies classification in 3D seismic data volume of the Brazilian Pre-salt through convolutional neural networks technology. CNN achieved relevant results in the characterization of seismic facies of Brazilian Pre-salt oil reservoirs. The average accuracy for all wells presented to CNN was $98 \%$ in average, an extraordinarily high value that ensures that facies prediction using CNN technology is a safe method for facies classification purposes.

\section{Acknowledgments}

Invision Geophysics thanks Repsol-Sinopec Brasil research group, especially Leonildes Soares, Cassiane Nunes and Támara Garcia for all support to this work and Dr. Luiz Braga, Murilo Lacerda and Uilli Freitas for collaboration and valuable discussions.

This project is a joint research effort of Repsol-Sinopec and Invision Geophysics, developed with the ANP research and development incentive law $\mathrm{n}^{\circ}$ 9.478, 06/08/1997. 


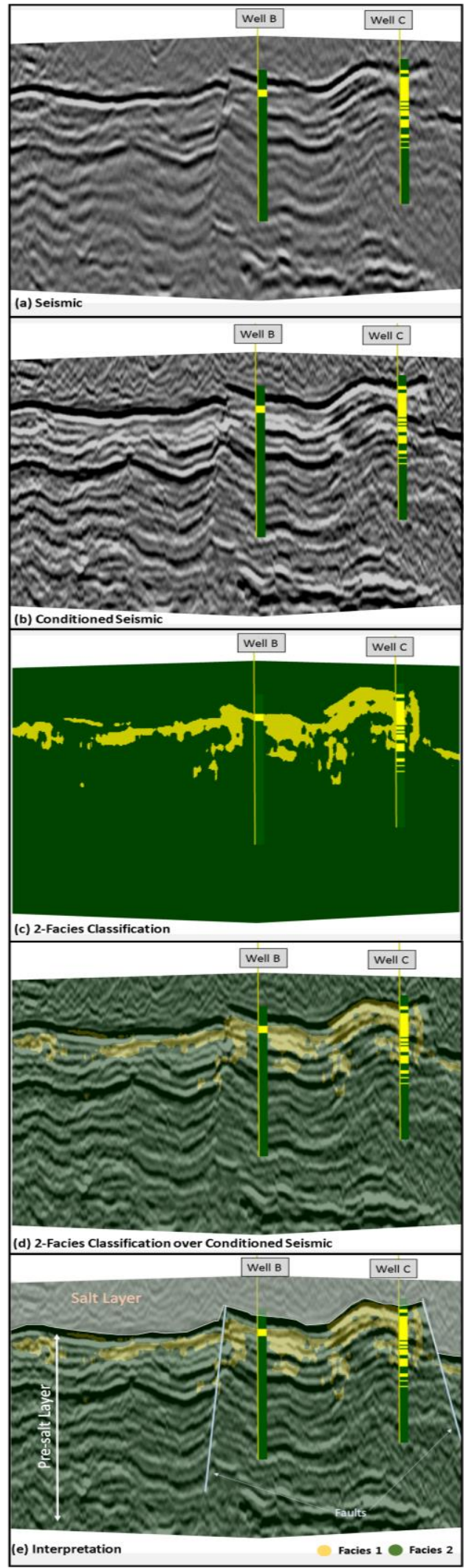

Figure 10 - Random line crossing two wells of the Brazilian Pre-salt (a) seismic; (b) conditioned seismic; (c) 2-Facies classification result - prediction; (d) 2-Facies classification over conditioned seismic and (e) preliminary interpretation of Pre-salt layer highlighting the main faults

\section{References}

Braga, I. L. S. Técnicas Multiespectrais Aplicada a fluxos de Inversão e Caracterização de Reservatórios de Hidrocarbonetos. Doctoral Thesis, UENF/LENEP 2011.

Braga, I. L. S.; Moraes, F. S. High-resolution Gathers by Inverse Q Filtering in the Wavelet Domain. Geophysics, Vol. 78, No. 2, V53-V61, 2013.

Goodfellow, I.; Bengio, Y.; Courville, A. Deep Learning: Adaptive Computation and Machine Learning. The MIT Press, Cambridge, Massachusetts. 775 p., 2016.

Hodgkin, A. L.; Huxley, A. F. A Quantitative Description of Membrane Current and its Application to Conduction and Excitation in Nerve. Bulletin of Mathematical Biology, Vol. 52, No. 1/2, p. 25-71, 1990.

LeCun, Y. et al. Handwritten Digit Recognition: Applications of Neural Network chips and Automatic Learning. IEEE Communications Magazine. p. 41-46, 1989.

McCulloch, W.; Pitts, W. A Logical Calculus of the Ideas Immanent in Nervous Activity. Bulletin of Mathematical Biophysics, Vol. 5, p. 115-133, 1943.

Da Silva, I. N. et al. Artificial Neural Networks: A Practical Course. Springer International Publishing, Switzerland. 307 p., 2017.

Clarifai. How does Clarifai's visual recognition API work? Available in: <https://www.clarifai.com/technology/> Accessed on: November 27, 2017. 